$12-1-1987$

\title{
Numerical Methods in Optics: A Course About Learning Physics Through Computing
}

James A. Lock

Cleveland State University, j.lock@csuohio.edu

Follow this and additional works at: https://engagedscholarship.csuohio.edu/sciphysics_facpub

Part of the Physics Commons

How does access to this work benefit you? Let us know!

\section{Publisher's Statement}

Copyright 1987 American Association of Physics Teachers. The article appeared in American Journal of Physics 55 (1987): 1121-1125 and may be found at http://aapt.scitation.org/doi/ 10.1119/1.15257

\section{Original Citation}

Lock, James A. "Numerical Methods in Optics: A Course About Learning Physics Through Computing." American Journal of Physics 55 (1987): 1121-1125.

\section{Repository Citation}

Lock, James A., "Numerical Methods in Optics: A Course About Learning Physics Through Computing" (1987). Physics Faculty Publications. 21.

https://engagedscholarship.csuohio.edu/sciphysics_facpub/21

This Article is brought to you for free and open access by the Physics Department at EngagedScholarship@CSU. It has been accepted for inclusion in Physics Faculty Publications by an authorized administrator of EngagedScholarship@CSU. For more information, please contact library.es@csuohio.edu. 


\section{Numerical methods in optics: A course about learning physics through computing}

James A. Lock

\section{INTRODUCTION}

It often happens in physics that basic physical laws are mathematically expressed in a simple and concise manner but their application to realistic situations results in lengthy and complicated mathematics. Technically oriented students who do not have a well-developed intuition for relating abstract mathematics to concrete situations can benefit from using computational methods as a learning tool. A course entitled Numerical Methods in Optics, developed at Cleveland State University, involves using the computer as a tool in helping the student to understand optical phenomena.

The choice of optics as the subject matter of the course was made for a number of reasons. First, if a student is going to program a physical phenomenon or a mathematical equation and get numbers as a final output, he must be confident that his program is correct and that his final numbers make sense. Two ways for a student to build such confidence are for his final numbers to agree with various limiting cases or with his intuition of how things should work out. But a more graphic way for him to believe in his computation is for the student to actually observe the phenomenon and compare his final numbers to what he sees. The subject of optics is special in this regard. The student can actually look at the caustic or reflection or diffraction pattern that he is trying to calculate. Observing the phenomenon also helps the student determine what types of graphical displays of the numerical results might be most effective. Second, it often happens in optics that if one is going to perform a numerical calculation of a phenomenon, the way of thinking about the problem at the outset and one's approach to it are different than if one were going to perform an analytical calculation. Examples of these alternative approaches are given in Sec. II. Optics demonstrates the concept of different but equivalent starting points to a problem very well.

The course was given in a ten-week quarter with the class meeting for three 65-min sessions per week. As prerequisites, students were expected to have taken a year of calculus, a year of calculus-based physics, and to know how to program in at least one computer language. The target 
group for this course consisted primarily of majors in computer science, engineering, and physics who had been exposed to optics in the calculus-based introductory course. The university computer laboratories were made available to the students. Each laboratory work station consists of an IBM-AT that supports the languages BASIC, PASCAL, and C. The graphics capabilities for BASIC and C are internal in the languages. The graphics capabilities for PASCAL are in TURBO GRAPHIC TOOLBOX which was available to the students. LOTUS 123 with its graphics capabilities was also available.

The course content of Numerical Methods in Optics was similar to that of a standard undergraduate optics course. There were four main areas of study, (i) mirrors and lenses, (ii) thin films and interference, (iii) diffraction, and (iv) scattering. It seemed best to limit the topics to these four but to treat each of them in more depth than is normally done in a standard undergraduate optics course. This permitted enough time to detail the numerical approaches to the topics and compare them to the analytic approaches. For each topic, the students were asked to write programs that calculated the phenomenon in question and that reproduced what they had seen in prior demonstrations of it. They were asked to see how the behavior of each phenomenon depended on the values of the input parameters and were required to use computer graphics in displaying their results.

\section{EXAMPLES OF NUMERICAL PROBLEMS IN OPTICS}

In this section we describe four of the practical optical problems treated in the course. Each one of the four cannot easily be solved analytically. But each is readily amenable to solution on a computer without requiring sophisticated numerical methods or elegant programming techniques. Each problem helps build one's intuition about how optical phenomena work and the results of each problem are easily comparable to demonstrations that the students observed. A complete list of the topics covered in the course is available from the author upon request.

\section{Reflection caustics from a cylindrical mirror}

The bright shimmering light patterns at the side of a swimming pool are portions of the caustics produced by the reflection of light from the curved surfaces of ripples and waves in the water. 'Similar caustics are seen in the bottom of a coffee cup, ${ }^{2}$ in a ring placed on a table, or on a piece of paper placed in a cake pan when these cylindrical reflecting surfaces are illuminated by diagonally incident light. The theory of caustics is well developed and an introduction to their mathematical categorization is given in Ref. 3. However, one can use a computer to understand the physical picture of how caustics are produced. A cluster of evenly spaced parallel light rays strike a curved mirror and are reflected. One's intuition suggests that the brightness of the reflected light at any point is related to the density of reflected light rays there. This is the case for the focal spot. All the reflected light rays pass through that point making it appear very bright. But is this intuition about the density of reflected rays correct at locations away from the focal point? By using the computer to follow the paths of the reflected light rays and to plot a dot-density diagram of the rays as they cross through some plane, one can verify whether this intuition linking the density of reflected rays to observed brightness is correct.

To analyze the reflected light rays mathematically, we consider a cylindrical surface of radius $a$ parallel to the $z$ axis given by the equation

$$
x=a-\left(a^{2}-y^{2}\right)^{1 / 2}
$$

and incident light rays parallel to the $x z$ plane making an angle $\theta$ with respect to the $x$ axis. If a ray strikes the cylinder at the point $\left(x_{0}, y_{0}, z_{0}\right)$ and reflects from it in the plane of incidence with the angle of reflection equal to the angle of incidence, one can calculate that the reflected ray crosses the $x y$ plane at the coordinates

$$
\begin{aligned}
x_{c}= & a\left\{1-\left[1-\left(\frac{y_{0}}{a}\right)^{2}\right]^{1 / 2}-\frac{z_{0}}{a} \cot \theta\right. \\
& \left.-\left(\frac{y_{c}}{y_{0}}-1\right)\left[1-\left(\frac{y_{0}}{a}\right)^{2}\right]^{1 / 2}\right\} \\
y_{c}= & y_{0}\left\{1+\frac{2 z_{0}}{a} \cot \theta\left[1-\left(\frac{y_{0}}{a}\right)^{2}\right]^{1 / 2}\right. \\
& \left.+\frac{\left(y_{0} / a\right)^{2} \cos ^{2} \theta+\sin ^{2} \theta}{\left(y_{0} / a\right)^{4} \sin ^{2} \theta-\left(y_{0} / a\right)^{2}-\sin ^{2} \theta}\right\}
\end{aligned}
$$

In order to numerically display the density of these points $\left(x_{c}, y_{c}\right)$ in the horizontal plane, we consider a cluster of incident rays that strike the cylindrical surface at many evenly spaced values of $y_{0}$ between $-a$ and $a$ and at many evenly spaced values of $z_{0}$ between 0 and the height of the cylinder $H$. The dot-density diagram in the horizontal plane is known as the spot diagram for the light reflected from the cylinder. Such a spot diagram is shown in Fig. 1. The plotted points to the left of the surface are the rays that in actuality would have reflected from the surface at least twice before crossing the $x y$ plane. It appears identical to the caustic observed in the bottom of a coffee cup and agrees with the intuition associating the density of reflected rays with the observed light intensity. The point of the cusp is the remnant of the focal point for normally incident

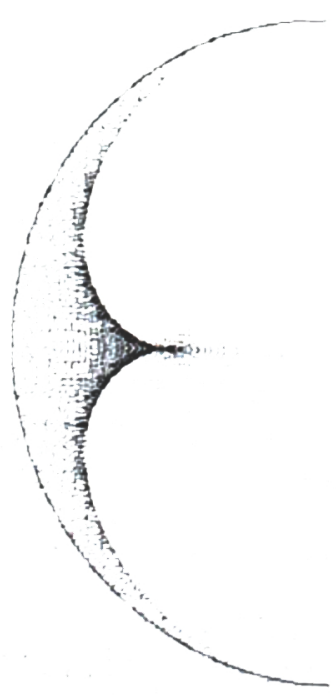

Fig. 1. The reflection caustic from a cylindrical surface. The angle of incidence is $45^{\circ}$, the height of the cylinder is equal to its diameter, and there are 9600 evenly spaced rays in the initial cluster. 
plane waves and the two curved portions of the cusp represent the fact that a mirror with a circular cross section does not have the perfectly focusing shape. But why are the regions of maximum intensity adjacent to regions of low intensity? This is answered in the next example.

\section{Aberrations of thick lenses}

Thin spherical lenses focus light very well. But thick spherical lenses are plagued by a number of aberrations. These can be demonstrated by trying to focus the light from a distant source with a large, thick magnifying glass. When the paraxial rays are focused, the edge rays are not. A similar effect is demonstrated by examining the focusing of sunlight incident upon a glass of water. ${ }^{2}$ For thin lenses, the analytical treatment of the refraction occurring at the front and rear surfaces of the lens is simple. ${ }^{4}$ For thick lenses, the only simple approach to the refraction is a $2 \times 2$ matrix raytracing method. ${ }^{5}$ The two variables in the ray-tracing approach are the height of a ray above the lens axis and its angle with the horizontal. A $2 \times 2$ matrix transfers these variables from one side of an air-lens interface to the other. Another $2 \times 2$ matrix transfers these variables between two adjacent interfaces, i.e., through the air or through the lens.

The $2 \times 2$ matrix ray-tracing method is easily implemented on a computer and is standardly used in multicomponent lens design. ${ }^{6}$ However, applied to a single converg. ing lens, it offers a very effective display of lens aberrations and their dependence on the lens thickness. For incident plane waves along the axis of the lens, the matrix ray-tracing method gives the results of Fig. 2 for a lens whose thickness is about $27 \%$ of its diameter. The separate focusing of the edge rays and paraxial rays, the circle of least confusion, and the caustic are readily evident in the figure and are easily observed in demonstrations. The appearance of the caustic in Fig. 2 answers the question posed at the end of Sec. II A. In Fig. 2 one observes the entire refracted rays whereas in Fig. 1 one observes the reflected rays in only one cross-sectional plane. Now one can see that the edge rays cross each other at a very shallow angle. This produces a long region where the rays almost sit on top of each other next to a region where there are no rays at all. This is why the caustic is bright and the region next to it is dark. The caustics of Fig. 2 can also be related to the caustics associat-

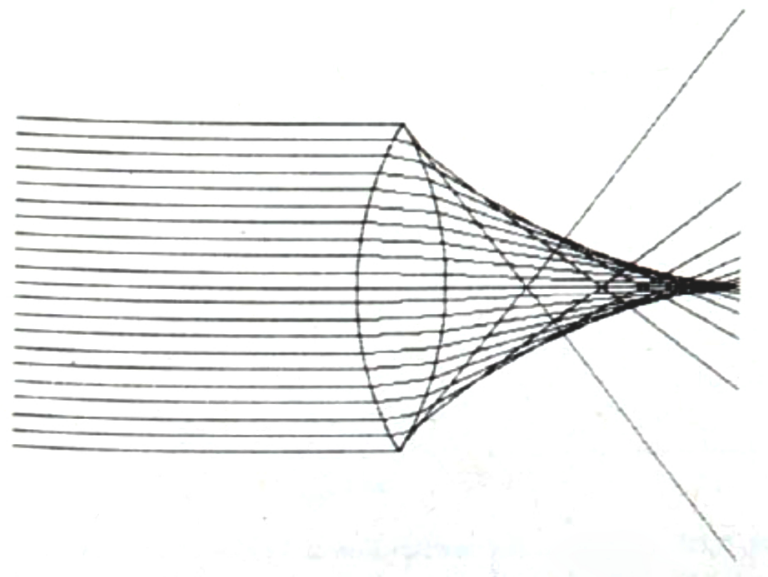

Fig. 2. The focusing properties of a lens whose thickness is 0.268 of its diameter. The index of refraction of the lens is $n=1.5289$. ed with sonic booms or the bow shock produced by boats in water. The physical mechanism responsible for producing the sonic boom or bow shock caustics is of course different. But the geometrical construction of the caustic is the same in each case.

\section{Multilayer thin films}

The colors on a soap bubble or between two plexiglass slabs pressed together are produced by thin-film interference. The equations describing reflection or transmission of normally or diagonally incident light on a thin film are not difficult to obtain. The interference of light from a stack of many thin films produces many impressive effects from the irridescent colors of abalone shells, peacock feathers, and morpho butterflies, ${ }^{7}$ to antireflection films on camera and binocular lenses, ${ }^{8}$ to the highly reflective dielectric mirrors used as the reflecting shrouds of projector lamps. The easiest way to analyze the reflective properties of multilayer thin films is a $2 \times 2$ matrix method that is derived from the boundary condition matching of the electric and magnetic fields at each of the interfaces. Each $2 \times 2$ matrix either transmits the forward- and backward-moving moving fields across an interface ${ }^{10}$ or across an entire coating. ${ }^{11}$ The effect of many thin films can be determined by multiplying together the appropriate number of $2 \times 2$ matrices. This can be easily accomplished on a computer. The elements of the matrices for this situation are in general complex numbers. If the software system one uses does not have complex arithmetic available, then the multiplications of the real and imaginary parts of the matrix elements must be explicitly carried out.

It is not hard to show that if one has a single layer of a high index of refraction material $(H)$ that separates two regions of low index of refraction $(L)$, or a single $L$ layer between two $H$ regions, the maximum reflection occurs when thickness of the single layer is a quarter wavelength of the light within the layer. One might suspect that this is also the case for a $H L H L \ldots . . H L H$ multilayer film on an $L$ substrate. This intuition of what layer thickness is responsi-

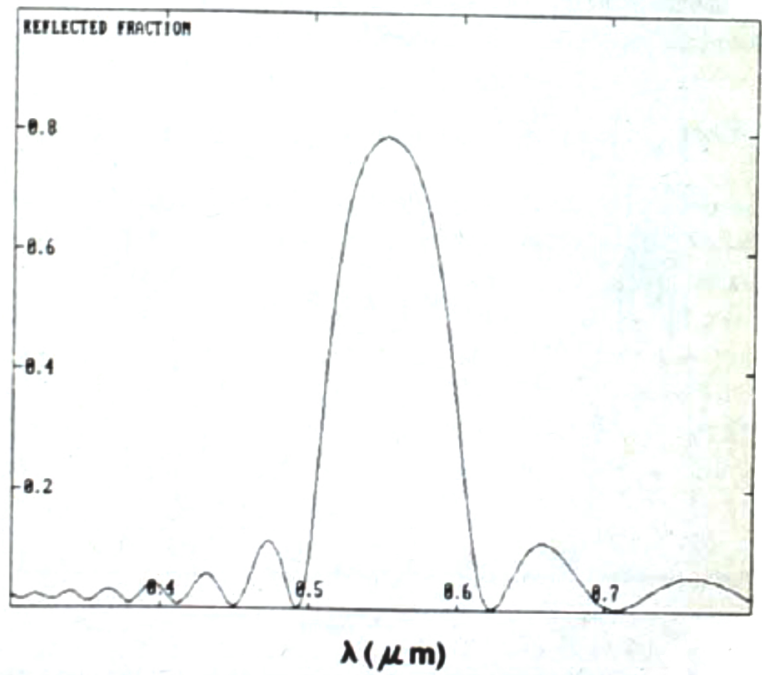

Fig. 3. Reflected intensity as a function of wavelength from a 17-layer stack of quarter-wave films on hydrated tissue. The indices of refraction of the layers alternate between $n=1.43$ and $n=1.23$. The hydrated tissue has an index of $n=1.33$. Such a graph describes the irridescent color of an insect wing casing. 


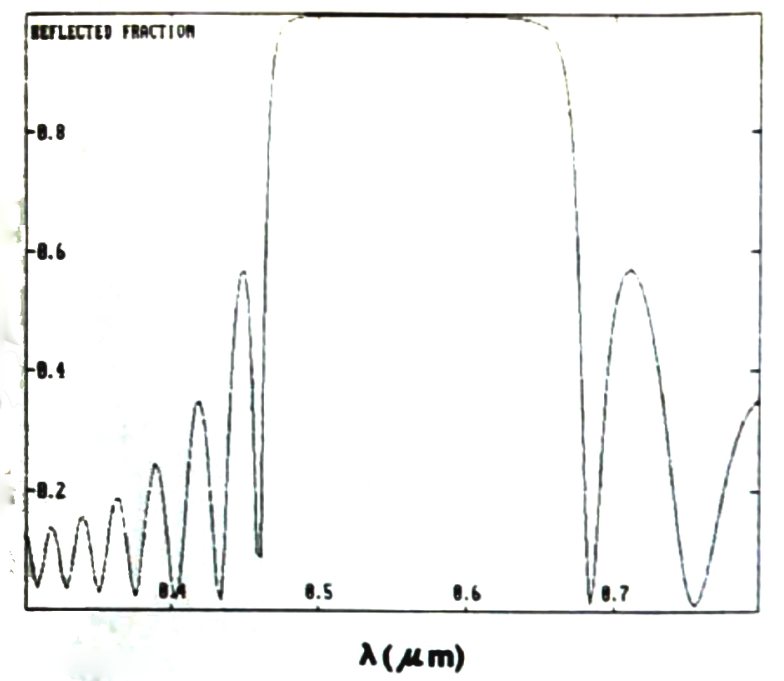

Fig. 4. Reflected intensity as a function of wavelength from a 17-layer stack of quarter-wave films on glass. The indices of refraction of the layers alternate between $n=2.32(\mathrm{ZnS})$ and $n=1.38\left(\mathrm{MgF}_{2}\right)$. The glass has $n=1.5289$. Such a graph describes a wide bandpass dielectric mirror.

ble for a bright reflection is verified in Figs. 3 and 4, which correspond to a single irridescent reflected color and a wide bandpass dielectric mirror, respectively.

\section{Fresnel diffraction from two-slit apertures}

In the last century, several clever geometric constructions such as the Cornu spiral and the Fresnel zone plates have been invented in order to help one understand the behavior of Fresnel diffraction in a number of cases such as the single-slit aperture, the straight edge, and the circular aperture on axis. However, these constructions do not help one to quantitatively calculate other Fresnel diffraction problems of interest such as the circular aperture off axis or

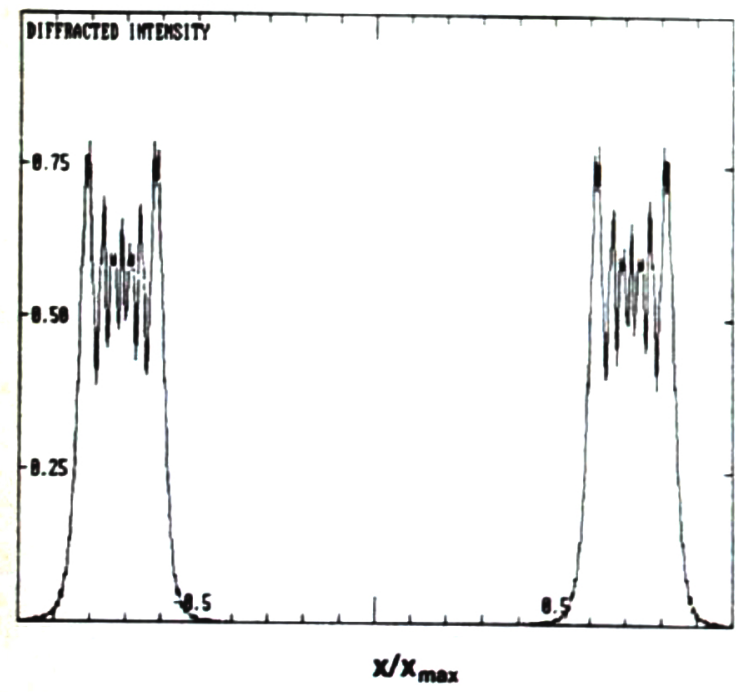

Fig. 5. Diffracted intensity as a function of position on the viewing screen from two-slit apertures of widths $0.5 \mathrm{~mm}$ whose inside edges are separated by $2.0 \mathrm{~mm}$ and which are illuminated with normally incident plane waves of $\lambda=0.55 \mu \mathrm{m}$. The viewing screen is $0.016 \mathrm{~m}$ away and the distance from center to edge on the viewing screen is $x_{\max }=1.75 \mathrm{~mm}$. These parameters correspond to the geometric shadow region.

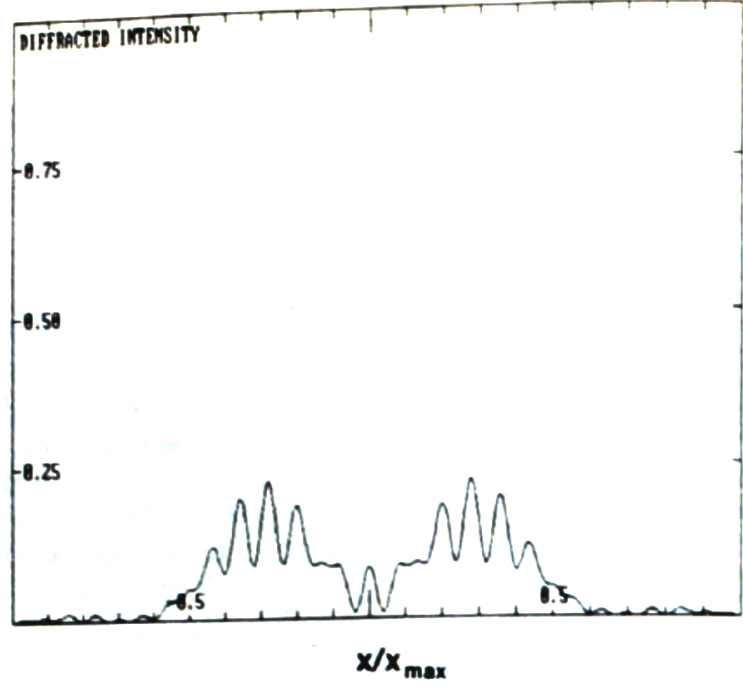

Fig. 6. Diffracted intensity as a function of position on the viewing screen for the two slits of Fig. 5. The viewing screen is $1.62 \mathrm{~m}$ away and the distance from center to edge on the viewing screen is $x_{\max }=4.44 \mathrm{~mm}$. These parameters correspond to the Fresnel transition region between the geometric and Fraunhofer limits.

the case of two-slit apertures. This latter class of problems is readily soluble numerically since rational function approximations and iterative methods may be used to rapidly calculate Fresnel integrals and their auxiliary functions. $^{12-14}$

The two-slit problem is of particular interest in this regard. One's intuitive feel is that very close to the apertures, the light intensity pattern is just the geometrical projection of each of the sources. Very far from the apertures, it is the standard two-slit Fraunhofer diffraction pattern since the diffracted field from each of the apertures completely overlaps the other and interferes with it. But somewhere between these two limits, the diffracted fields from the two slits should only partially overlap. The central portion of the total field, where the overlap occurs, should resemble

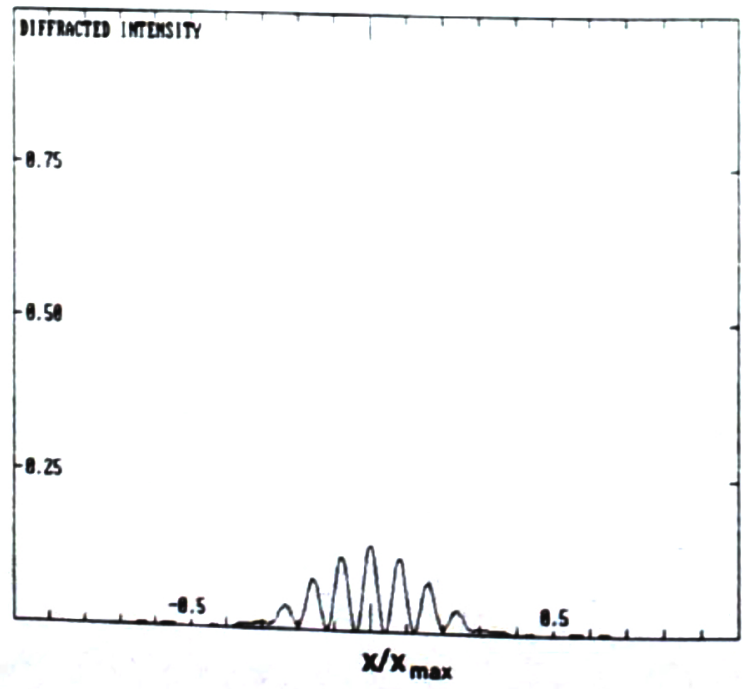

Fig. 7. Diffracted intensity as a function of position on the viewing screen for the two slits of Fig. S. The viewing screen is $6.46 \mathrm{~m}$ away and the distance from center to edge on the viewing screen is $x_{\max }=17.8 \mathrm{~mm}$. These parameters correspond to the Fraunhofer diffraction limit. 
the two-slit Fraunhofer interference pattern and the periphery of the total field, where the two fields have not overlapped, should resemble the Fresnel diffraction pattern from a single slit. On a computer one can continuously move the viewing screen outward from the sources and watch the transition take place.

We consider two slits each of width $2 a$ whose interior edges are separated by the distance $2 d$. The diffracted electric field on a viewing screen a distance $D$ from the aperture is

$E(x)=\left(i E_{0} / 2\right)[\mathscr{E}(\alpha)-\mathscr{E}(\beta)+\mathscr{E}(\gamma)-\mathscr{E}(\delta)]$,

where

$$
\mathscr{B}(x) \equiv C(x)+i S(x),
$$

where $C$ and $S$ are the Fresnel cosine and sine integrals, respectively,

$$
\begin{aligned}
& \alpha=\left(\frac{2 a^{2}}{\lambda D}\right)^{1 / 2}\left(\frac{x}{a}+\frac{d}{a}+2\right), \\
& \beta=\left(\frac{2 a^{2}}{\lambda D}\right)^{1 / 2}\left(\frac{x}{a}+\frac{d}{a}\right), \\
& \gamma=\left(\frac{2 a^{2}}{\lambda D}\right)^{1 / 2}\left(\frac{x}{a}-\frac{d}{a}\right), \\
& \delta=\left(\frac{2 a^{2}}{\lambda D}\right)^{1 / 2}\left(\frac{x}{a}-\frac{d}{a}-2\right),
\end{aligned}
$$

and where $x$ is the distance from the center of the viewing screen. The diffracted intensity is the magnitude of $E(x)$ squared. Using the values of $C$ and $S$ obtained by the rational function approximation to auxiliary Fresnel functions, and the parameters $\lambda=0.55 \mu \mathrm{m}, 2 a=0.5 \mathrm{~mm}, 2 d=2.0$ mm, Figs. 5-7 show the Fresnel diffracted intensity close to the apertures, in the transition zone, and in the Fraunhofer limit, respectively.

\section{STUDENT ASSESSMENT}

In the spring 1986 quarter, two physics majors, two computer science majors, a mathematics major, a mechanical engineering major, and the physics department equipment technician enrolled in Numerical Methods in Optics. Their programming experience was primarily with BASIC and PASCAL. Students were given about $1 \frac{1}{2}$ weeks to write each program. At the end of the course, students completed written evaluations and found the course to be a very positive experience. They had been used to courses that spent more time on formalism generation and analytic calculations as homework. The students commented that (1) it was novel and interesting to set up realistic situations on a computer instead of using analytical methods only, (2) they spent more time on the homework than in other courses but they enjoyed the computer-based problems, (3) actually having to program the general theory to solve a specific problem helped them understand and remember the basic principles and equations, (4) they were forced to be very specific in choosing values for input parameters in order to obtain realistic and reasonable results, and (5) they enjoyed playing with the input values to see how the computer graphics pictures changed when the input was varied. The students who were already familiar with programming said that they learned nothing new about computing or programming from the course. One student without much previous experience said that he learned a lot about programming. One other student commented that he became aware that computers could be used for more than running canned software.

These student comments lead the author to believe that the computational approach to homework was effective in helping the students both learn optics and make the mathematics look real to them. It is reasonable to expect that such an approach might also be successful in other advanced physics courses such as classical mechanics, electricity and magnetism, and thermodynamics.

\section{ACKNOWLEDGMENT}

The author would like to thank the referees for several helpful comments on an earlier version of this manuscript.

'D. Falk, D. Brill, and D. Stork, Seeing the Light (Harper and Row, New York, 1986), p. 85.

${ }^{2}$ Reference 1, p. 98.

${ }^{3}$ J. Walker, Sci. Am. 249, 190 ( 1983 ).

${ }^{4}$ D. Halliday and R. Resnick, Fundamentals of Physics (Wiley, New York, 1981 ), 2nd ed., extended version, Chap. 39

${ }^{5}$ A. Nussbaum and R. A. Phillips, Contemporary Optics for Scientists and Engineers (Prentice-Hall, Englewood Cliffs, NJ, 1976), Sec. 2.2.

${ }^{6}$ E. Hecht and A. Zajac, Optics (Addison-Wesley, Reading, MA, 1979), pp. 175-176

'K. Nassau, The Physics and Chemistry of Color (Wiley, New York, 1983), pp. 265-268

${ }^{8}$ Reference 5, pp. 191-193

'Reference 6, pp. 315-316

${ }^{10} \mathrm{~J}$. R. Reitz, F. J. Milford, and R. W. Christy, Foundations of Electromagnetic Theory (Addison-Weslcy, Reading, MA, 1979), 3rd ed., problem 18-12.

"Reference 6, Sec. 9.9.1.

${ }^{12}$ B. R. Frieden. The Computer in Optical Research (Springer, New York, 1980), Sec. 2.1.

${ }^{13}$ Handbook of Mathematical Functions, edited by M. Abramowitz and 1 A. Stegun (National Bureau of Standards, Washington, DC, 1964), Sec. 7.3 .

${ }^{14}$ M.A. Heald. Am. J. Phys 54, 980 (1986).

Post-print standardized by MSL Academic Endeavors, the imprint of the Michael Schwartz Library at Cleveland State University, 2017. 